\title{
Spin-Rotation Coupling in Ferromagnetic Clusters
}

\author{
G.F. Bertsch and V.Visuthikraisee \\ Dept. of Physics and Institute for Nuclear Theory \\ University of Washington \\ Seattle, WA 98195
}

\begin{abstract}
We examine the magnetic response of free clusters considering the spin direction and the cluster orientation as the only active degrees of freedom. The average magnetization in small fields approaches the Langevin value for paramagnets, depending on the degree to which the Hamiltonian preserves symmetries. Superparamagnetic behavior is not achievable within models considering only these degrees of freedom.
\end{abstract}

Typeset using REVTEX 


\section{INTRODUCTION}

Experimental studies of magnetism of free atomic clusters have shown a number of interesting phenomena [1] 5,?,7]. Susceptibilities measured in Stern-Gerlach experiments can be large, with an irregular dependence on the number of atoms in the cluster. High susceptibilities are found not only in normally ferromagnetic elements but also in an element, rhodium [8], which is not ferromagnetic in the bulk. The character of the susceptibility is

also variable. In many cases it is described phenomenologically as superparamagnetism, which means that besides a large susceptibility the clusters behave uniformly, independent of initial state within the thermal ensemble. The clusters may also respond with a large variance in their magnetization, with some clusters even having magnetic moments opposite the field. Of course, the limiting behavior of this kind is the magnetization distribution of an isolated atom, proportional to the spin projection along the magnetic field.

The general theoretical problem we address is whether the observed behavior of free magnetic clusters can be understood in the framework of Hamiltonians that treat only rotational and spin degrees of freedom, neglecting vibrations. Vibrational coupling is of course important for the response of warm clusters [4], but the question of whether cold clusters can show superparamagnetism is still open. This paper is an extension of earlier work which considered a very simplified Hamiltonian with only rotational degrees of freedom active [20,10]. Large average susceptibilities were found to be possible, but not superparamagnetism.

We shall calculate the cluster magnetism in a quantum mechanical representation, although the angular momenta are high enough that classical methods should suffice. The rotor is represented in the usual basis $(J K M)$, with $M$ the component of angular momentum about the $z$ axis and $K$ the component about the intrinsic third axis. The rotor is coupled to spin states in the basis $\left(S S_{z}\right)$. Taking the field direction along the $z$ axis, the component of angular momentum about that axis, $M+S_{z}$, is conserved. In the earlier studies, the simplified Hamiltonian conserved $K$ as well.

The theoretical magnetic response associated with a particular Hamiltonian is most 
conveniently discussed by comparing with Langevin model of paramagnetism. Here the spin is coupled to a nonspecific heat bath to give the following formula for the magnetization in an external field,

$$
\begin{aligned}
\left\langle\mu_{z}\right\rangle & =\frac{\int_{-\mu_{0}}^{\mu_{0}} d \mu_{z} \mu_{z} \exp \left(\mu_{z} B / k T\right)}{\int_{-\mu_{0}}^{\mu_{0}} d \mu_{z} \exp \left(\mu_{z} B / k T\right)} \\
& =\mu_{0}\left(\operatorname{coth}(x)-\frac{1}{x}\right)
\end{aligned}
$$

Here $\mu_{0}$ is the intrinsic magnetic moment and the parameter $x$ is the ratio of magnetic energy to thermal energy, $x=\mu_{0} B / k T$. This formula is sometimes used to deduce intrinsic magnetic moments from the observed deflections in the Stern-Gerlach apparatus. At low field strength the Langevin formula has the limiting behavior,

$$
\frac{\left\langle\mu_{z}\right\rangle}{\mu_{0}}=\frac{x}{3}
$$

The condition $x \ll 1$ is in fact satisfied for the conditions of the cited experiments, which involved clusters of a few tens or hundreds of atoms.

Given the Hamiltonian, the magnetic response is calculated as follows. First, the Hamiltonian is diagonalized for a range of magnetic field strengths to produce a spectrum of eigenenergies $E_{i}(B)$. The magnetic moments of the states are then calculated using the formula

$$
\mu_{i}(B)=\left\langle i B\left|\mu_{z}\right| i B\right\rangle=-\frac{d E_{i}}{d B}
$$

Since eq. (四) does not require the eigenfunctions, a substantial savings of computation time is possible with respect to a full diagonalization. The distribution of eigenvalues is given by the expression

$$
P(\mu)=\frac{\sum_{i}^{N} P_{i} \delta\left(\mu_{i}-\mu\right)}{\sum_{i}^{N} P_{i}} .
$$

Here $N$ is the dimensionality of the Hamiltonian and $P_{i}$ are the probabilities of the states. We take the adiabatic limit by assigning the probabilities as the Boltzmann factors of the levels in the absence of a magnetic field, 


$$
P_{i}=\exp \left(-E_{i}(0) / k T\right)
$$

In applying this, the levels are always ordered and indexed by energy. Thus the lowest level in zero field sets the Boltzmann factor for the lowest level at other field strengths. This assumes that all level crossings are avoided crossings. It does appear for the Hamiltonians we study that the only true level crossings are those imposed by symmetry ( $M$ and possible $K)$. We do not address the question of whether the field changes take place slowly enough for the adiabatic assumption to be valid at the avoided level crossings.

\section{HAMILTONIAN}

We consider a Hamiltonian model in which the spin orientation is coupled to an intrinsic axis of the cluster and to an applied field $B$. The Hamiltonian can be written as

$$
H=\sum_{i} \frac{J_{i}^{2}}{2 I_{i}}-\frac{\lambda}{S^{2}-S / 2} \sqrt{\frac{4 \pi}{5}} \sum_{m} Y_{2 m}^{\dagger} s_{2 m}-s_{z} \mu_{0} B
$$

where $I_{i}$ 's are the moments of inertia. The second term is the anisotropy energy, the coupling of the spin to the intrinsic axis of the cluster. We assume a uniaxial anisotropy having the

form of an $L=2$ tensor, which would apply to clusters of low symmetry. A Hamiltonain of this form was also considered in ref. [11]. The usual anisotropy energy applying to cubic crystals has the form of an $L=4$ tensor.

The dimensionalities of the state vector spaces for cluster spin-rotor wave functions require us to make additional simplifications. The angular momenta present in these clusters are of the order of 1000 for a cluster of 100 iron atoms at room temperature. It is impractical from a numerical standpoint to deal with ensembles having such large angular momenta. Since the quantization of angular momentum should not play a role at large values, we believe it is acceptable to use a smaller basis, scaling the parameters of the Hamiltonian and the temperature appropriately. Even with a smaller basis, the full Hamiltonian still gives large matrices, so we consider as well a limiting case where the spin is not an independent dynamical variable, but is fixed to the cluster axis, the "locked-spin" model. 
We further assume in the locked-spin model that the cluster has two equal moments of inertia, which we take as the 1 - and 2- axes, $I_{1}=I_{2}$. Then in the usual $|J K M\rangle$ basis $K$ is conserved by the rotational Hamiltonian. Its nonvanishing matrix elements are the diagonal elements, given by

$$
\left\langle J K M\left|\sum_{i} \frac{J_{i}^{2}}{2 I_{i}}\right| J K M\right\rangle=\frac{J(J+1)}{2 I_{1}}+\frac{\left(I_{1}-I_{3}\right) K^{2}}{2 I_{1} I_{3}}
$$

A fully asymmetric rotor requires an additional term in the Hamiltonian with matrix elements proportional to

$$
\left\langle J K+2 M\left|J_{1}^{2}-J_{2}^{2}\right| J K M\right\rangle=\sqrt{(J(J+1)-K(K+1))(J(J+1)-(K+1)(K+2))}
$$

We will briefly consider this term later.

The rotor is coupled to the magnetic field through the spin, whose orientation with respect to the intrinsic 3 -axis is denoted by $\theta$. The matrix elements of the magnetic field term in the Hamiltonian are diagonal in $M$ but not necessarily in $J$ or $K$. They are given by

$$
\begin{aligned}
\left\langle J K M\left|\mu_{z} B\right| J^{\prime} K^{\prime} M^{\prime}\right\rangle= & \delta_{M, M^{\prime}} \mu_{0} B \sqrt{\frac{2 J^{\prime}+1}{2 J+1}}\left\langle J^{\prime} M 10 \mid J M\right\rangle \\
& \times\left\{\cos \theta\left\langle J^{\prime} K^{\prime} 10 \mid J K\right\rangle+\frac{\sin \theta}{\sqrt{2}}\left[\left\langle J^{\prime} K^{\prime} 1-1 \mid J K\right\rangle-\left\langle J^{\prime} K^{\prime} 11 \mid J K\right\rangle\right]\right\} .
\end{aligned}
$$

The more general Hamiltonian allowing intermediate coupling of the spin is constructed in the $\left|J K M S S_{z}\right\rangle$ basis. The magnetic energy is trivial in this basis. It depends only on $S_{z}$, with the matrix element $\left\langle S_{z}\left|\mu_{0} B\right| S_{z}\right\rangle=\mu_{0} B S_{z}$. The anisotropy energy is given by

$$
\begin{gathered}
\left\langle J K M S S_{Z}\left|\sum_{m} Y_{2 m}^{\dagger} s_{2 m}\right| J^{\prime} K^{\prime} M^{\prime} S S_{z}^{\prime}\right\rangle=(-1)^{2 S+S_{z}^{\prime}-S_{z}} \sqrt{\frac{5}{4 \pi}} \sqrt{\frac{(2 J+1)(S+1)(2 S+3) S(2 S-1)}{4\left(2 J^{\prime}+1\right)}} \\
\delta_{K, K^{\prime}} \delta_{M+S_{z}, M^{\prime}+S_{z}^{\prime}}\left\langle J M 2\left(M^{\prime}-M\right) \mid J^{\prime} M^{\prime}\right\rangle\left\langle J K 20 \mid J^{\prime} K\right\rangle\left\langle S S_{z} 2\left(M-M^{\prime}\right) \mid S S_{z}^{\prime}\right\rangle
\end{gathered}
$$




\section{RESULTS: LOCKED SPIN MODEL}

The locked-spin model for a spherical rotor was treated in ref. [2, 20, 10, 12]. In this model, $K$ is a conserved quantum number if the intrinsic 3 -axis is taken along the spin direction. The previous work showed that the adiabatic ensemble produces an average susceptibility in low fields of $2 / 3$ of the Langevin value. The distribution $P(\mu)$ is very broad, however, far from the idealized superparamagnet. A weak point of the model is its assumed $K$ conservation: one could argue $K$ conservation is too restrictive to allow adequate mixing of the spin with the other degrees of freedom. We shall find that breaking $K$ does in fact have a significant effect on the average magnetization, but not on the spread.

We first remark that changing the Hamiltonian by making the rotor deformed has negligible effect on the distribution, in the absence of $K$ mixing. When $K$ is conserved, the deformed rotor has the same wave function as the spherical rotor. The magnetic moments of the states are thus the same, only the occupation probabilities differ. The thermal ensemble would then favor states a somewhat different distribution of $K$ values, but the overall effect is small. This is illustrated with the moment distributions for the deformed symmetric rotor shown in Fig. 1. The curves were calculated with an ensemble having a cutoff angular momentum of 40 for the rotor. The temperature was fixed at $k T=100$ in units of $I_{1}^{-1}$ and the magnetic field strength has the same value, $\mu_{0} B=100 I_{1}^{-1}$. To display the distribution, eq. (5) was smeared with a Gaussian of the form $\exp \left(-\left(\mu-\mu_{i}\right)^{2} /(\Delta \mu)^{2}\right)$ with $\Delta \mu=0.05 \mu_{0}$. The different curves show results for Hamiltonians with moment of inertia ratios ranging from $1 / 2$ to 2 . One sees that there is hardly any change in the distribution of magnetic

moments. The average magnetization changes by less than $10 \%$ over this extreme range of deformations.

Without discussing in detail the $K$-mixing in the Hamiltonian, it is possible to see in a crude model how it affects the magnetization. We compute the energy with a Hamiltonian that conserves $K$, but combine the ensembles for different $K$ (but the same $M$ ) into a common ensemble in eq. (5). This assumes that the K-mixing makes all level crossings 
avoided crossings, but that it is not strong enough to affect the states between crossing points. This ensemble produces a higher average magnetization, as might be expected: the crossing of states of different $K$ allows the probability to jump to states of larger magnetic moment. In Fig. 2 we show the average magnetization of this mixed- $K$ ensemble compared with the Langevin and with the fixed- $K$ locked-spin model. One sees that the mixed- $K$ ensemble has a susceptibility close to the Langevin model for field strengths below $x=0.2$. However, the spread in moments is very similar to the fixed- $K$ ensemble.

We now discuss the behavior of eigenstates in Hamiltonians with mixed $K$. In the lockedspin models, the $K$ mixing can be induced by a tipped spin with $\theta$ nonzero, or by a fully asymmetric rotor with the additional term eq. (9) in the Hamiltonian. The effect of the tipped spin may be seen in the energy level plot in Fig. 3. The plot show the energies of the $M=1$ states with spin angle tipped by $5^{\circ}$ with respect to the symmetry axis. Several avoided level crossings may be seen. For example, the uppermost crossing involves a transition between states that have quantum numbers $(J K)=(20)$ and $(J K)=(1,-1)$ in zero field. We can see that the effect of the $K$-mixing is limited to regions very close to the level crossings; the spectrum is hardly changed otherwise. This means that the magnetic moments of the levels are unaffected except for special values of the field. The consequence is that the dispersion in magnetic moments is very similar to that of the fixed $K$ ensemble. This may be seen in Fig. 4, where we show the magnetization distribution for a range of tipping angles. There is very little difference between the different Hamiltonians. The average magnetization from the diagonalized Hamiltonians with mixed $K$ is also very close to the mixed- $K$ ensemble shown in Fig. 2.

Returning to the spread in magnetization distribution, it appears that the locked-spin Hamiltonian cannot explain superparamagnetic behavior. The one caveat in this statement is that we have not considered fully asymmetric Hamiltonians. The perturbation eq. (9) does not produce a large mixing of the wave functions, and seems unlikely to have a significant effect on the widths. 


\section{RESULTS: INTERMEDIATE COUPLING}

We now generalize from the locked-spin Hamiltonians to consider the spin as an independent degree of freedom. We shall treat the intermediate coupling spin-rotor Hamlitonian assuming that the inertia tensor is axially symmetric. As we saw in the last section, the breaking of $K$ symmetry has no qualitative effect on the magnetic response, but only changes somewhat the magnitude of the susceptibility coefficient. We study the ensemble with a maximum orbital angular momentum of $J_{\max }=30$ and the spin value $S=1$. Despite the modest size of these numbers, this space requires diagonalization of about 4000 matrices of dimensionality ranging up to about 100. The ensembles are generated with a temperature of $T=100 / I_{1}$ as before.

The first task is to see whether the locked-spin results can be reproduced with a spin $S$ as small as $S=1$. For this purpose we calculate the moment distribution taking a large value for the anisotropy coupling, $\lambda=5 \mu B$. Typical results are shown in Fig. 5. In plotting the distribution, the magnetic moments have been scaled so that the maximum is \pm 1 in either model. We see that the two curves are practically identical, giving us confidence in using the Hamiltonian with a small value for $S$. The moment distribution in a weak coupling situation, $\lambda=0.01 \mu_{0} B$, is shown in Fig. 6. Here the spin behaves as though it were uncoupled, so one sees the three $M$-states as in the atomic deflection. Notice however that the occupation probabilities are unequal. The probabilities are shifted at the level crossings, which become avoided crossings due to the (small) anisotropy term. At intermediate values of the coupling, the distribution changes smoothly from the three-peak structure in Fig. 6 to the typical locked-spin shape of Fig. 5. In all cases the distribution remains broad.

The field dependence of the magnetization shows a more complex behavior with intermediate coupling than we found for the locked spin. There are several distinct regions of behavior, depending on the relative sizes of the magnetic energy with respect to the anisotropy energy, and the rotational frequency with respect to the Larmor frequency. In extremely low fields, with magnetic energies less than the anisotropy energy, the spin should 
behave in a similar way to the locked-spin model. When the magnetic field energy becomes larger than the anisotropy energy, the spin decouples from the rotational motion and we should see the individual $S_{z}$ states as in Fig. 6. These two regimes are illustrated in Fig. 7, showing the magnetization distribution for $x=0.005$ and 0.02 for a model with weak coupling, $\lambda=0.01 k T$. The distribution in the lowest field, shown by the solid line, is broad with a peak at $\mu=0$. This is similar to the locked-spin behavior, $P(\mu) \sim \log \left(\mu / \mu_{0}\right)$. At $x=0.02$, where the magnetic field is twice the anisotropy energy, the distribution has already dissolved into the 3 -peak structure of the decoupled $S=1$ states. The behavior of the average magnetization as a function of $x$ is shown in Fig. 8. In the extreme low-field limit, $\mu_{0} B<\lambda$, the magnetization is close to the weak-field Langevin function, eq. (3). The spin decouples from the rotational motion when $B$ exceeds $\lambda$, as already mentioned. From Fig. 8 it may be seen that the average magnetization increases rather slowly between $\mu_{0} B \sim 0.01 k T$ and $0.08 k T$. Above that point the magnetization rises to even exceed the Langevin value. We believe that this change of behavior is associated with level crossings of the type $\left(J S_{z}\right) \rightarrow\left(J \pm 1 S_{z} \mp 1\right)$, corresponding to the matching of rotational and Larmor frequencies. A change of behavior at this matching point has been suggested previously [6.13].

It is interesting to examine the susceptibility in the third region in more detail. Here the magnetization is still far below the saturation condition $\mu_{0} B \sim k T$, and it is roughly linear in the field. We make a linear fit to the magnetization with the function,

$$
\mu=a \mu_{0} x
$$

The dependence of the fit coefficient $a$ on magnetic field is plotted in Fig. 9. We see that the behavior like the the locked-spin model only if the anisotropy energy is substantially above $k T$. Below that, the coefficient exceeds the Langevin value, $a=1 / 3$, as we saw on Fig. 7 .

Our findings for the magnetic response are quite different from those of ref. [1], where a similar Hamiltonian was considered. In that work, the magnetization was found to be independent of the temperature but to depend strongly on the anisotropy energy. However, 
these authors did not treat the adiabatic ensemble, but rather a mixed ensemble in which the magnetic field was turned on suddenly.

We conclude this section with an estimate of the magnitude of $\lambda$ for iron clusters. The cubic anisotropy constant $K$ for bulk iron is measured as [18] $K \approx 4 \times 10^{5} \mathrm{ergs} / \mathrm{cm}^{3}=5 \times 10^{-4}$ $\mathrm{eV} /$ atom. For a cluster of 20 iron atoms this is $2.5 \times 10^{-4} \mathrm{eV}$, which scaled to room temperature implies $\lambda \sim 0.01 k T$. Thus the weak coupling limit we discussed above should apply to moderate sized iron clusters at room temperature. A typical rotational frequency is given by $\left\langle J^{2}\right\rangle^{1 / 2} / I \sim(k T / I)^{1 / 2}$. This has a magnitude of about $10^{-4} \mathrm{eV} / \hbar$ for a cluster of 20 iron atoms, which is the order of magnitude of the Larmor frequency in a $1 \mathrm{~T}$ field. Thus, the model predicts that light mass iron clusters would behave with the spin nearly decoupled from the rotational motion and with a fairly weak average susceptibility. This is certainly contrary to experiment, which has never demonstrated atomic-like behavior in clusters larger than diatomic.

\section{CONCLUSION}

Our study of the magnetic response of rotors coupled to spin is guided by the question, to what extent can a thermal ensemble of rotors play the role of the thermal bath that one assumes in the Langevin model? One's intuition may be that the rotor has far too few degrees of freedom to serve as a heat bath. Nevertheless, the low-field magnetic susceptibility of the thermal bath is reproduced on average by the deformed rotor with a locked and tipped spin axis. It is also obtained for intermediate coupling of spin to the rotor, except in a small interval of field strengths.

However, superparamagnetism implies not only that the average moment is given by the Langevin formula, but all clusters should behave identically. Here the adiabatic rotor ensemble behaves quite differently, always having a large variance in the magnetization.

Let us ask now, what would be needed in the spin-rotor Hamiltonian to achieve superparamagnetism? Clearly there would have to be much stronger $K$ mixing than can be 
generated by the spin-rotor Hamiltonian. Recall in Fig. 3, the energy levels have different slopes with large stretches of constant moment separated by small intervals of the level crossings.

In the current idiom, we may speak of this as a weakly perturbed regular Hamiltonian. To achieve superparamagnetism, the Hamiltonian would have to be chaotic. Quantum mechanical chaos is a regularity and evenness in the energy spectra associated with the complete mixing of wave functions. In such a spectrum, the slopes of the levels would be similar and governed by the average behavior of the levels in that region of energy. When the Hamiltonian is chaotic, the slopes have universal correlation properties [16, 17]. This behavior would require additional terms in the Hamiltonian eq.(7). A minimum condition for chaotic behavior is that the off-diagonal matrix elements exceed the level spacing of the regular part of the Hamiltonian [14].

It is expected that the coupling of the spin to vibrations could provide the thermal bath that would produce superparamagnetism [19,20]. However, it has not been demonstrated that this mechanism actually would work in finite-size clusters. The first issue is that vibrational transitions would have to be at the same energy as the spin flip in the external field. This is to allow off-diagonal matrix elements to nearby levels. The maximum vibrational frequency has the order of magnitude of the Debye energy $\hbar \omega_{D}$; the spacing of vibrational levels $\Delta E_{v i b} \sim \hbar \omega_{D} / 3 N$, where $N$ is the number of atoms in the cluster. In a $1 \mathrm{~T}$ field, the spin flip energy is $10^{-4} \mathrm{eV}$. With a Debye energy in iron of $6 \times 10^{-3} \mathrm{eV}$, the vibrational level spacing is smaller than the spin-flip energy for clusters larger than $N=20$. Spin relaxation in the bulk takes place mainly through Raman scattering of phonons. In this case one is interested in the difference of two vibrational energies, and the vibrational level spacing again sets the scale. The next condition is that the average off-diagonal matrix element of the vibrational coupling should exceed the level spacing. This will always be satisfied for sufficiently large clusters, because $V$ and $\Delta E$ vary as $V \sim \sqrt{(\Delta E)}$ as the size of system varies. This follows from the requirement that the relaxation time is independent of the size of the system for large $N$. Unfortunately, the coupling is not well enough known at present 
to make a numerical estimate.

\section{ACKNOWLEDGMENT}

We acknowledge discussions with N. Onishi and A. Bulgac. This work was supported by the Dept. of Energy under Grant FG06-90ER40561. 


\section{REFERENCES}

[1] W.A. de Heer and P. Milani and A. Châtelain, Phys. Rev. Lett. 65 (1990) 488.

[2] J.P. Bucher and D.C. Douglass and L.A. Bloomfield, Phys. Rev. Lett. 66 (1991) 3052.

[3] D.C. Douglass and J.P. Bucher and L.A. Bloomfield, Phys. Rev. Lett. 68 (1992) 1774.

[4] I.M.L. Billas and J.A. Becker and W.A. de Heer, Z. Phys. D 26 (1993) 325.

[5] D.C. Douglass and D.M. Cox and J.P. Bucher and L.A. Bloomfield, Phys. Rev. B 47 (1993) 12874.

[6] I.M.L. Billas and J.A. Becker and A. Châtelain and W.A. de Heer, Phys. Rev. Lett. 71 number 24 (1993) 4067.

[7] S.E. Apsel and J.W. Emmert and J. Deng and L.A. Bloomfield, to be published

[8] A.J. Cox, et al., Phys. Rev. lett. 71 (1993) 923.

[9] G.F. Bertsch and K. Yabana, Phys. Rev. A49 (1994) 1930.

[10] G.F. Bertsch, N. Onishi, and K. Yabana, Z. Phys. D34 (1995) 213.

[11] P. Ballone, P. Milani and W.A. de Heer, Phys. Rev. B44 (1991) 10350.

[12] A. Maiti and L. Falicov, Phys. Rev. B48 (1993) 13596.

[13] P.J. Jensen and K.H. Bennemann, Z. Phys. D26 (1993) 246.

[14] M.R. Zirnbauer, J. Verbaarschot and H.A. Weidenmueller, Nucl. Phys. A411 (1983) 161.

[15] A.R. Edmonds, Angular Momentum in Quantum Mechanics, Princeton 1960.

[16] A. Szafer and B.L. Altshuler, Phys. Rev. Lett. 70 (1993) 587.

[17] D. Kusnezov and D. Mitchell, Phys. Rev. C, to be published.

[18] K.H. Hellwege, ed. Landolt-Boernstein Numerical Data and Functional Relationships in Science and Technology, vol. III/19a, (1981) 42. 
[19] S.N. Khanna and S. Linderoth, Phys. Rev. Lett. 67 (1991) 742.

[20] J.A. Becker, Z. Phys. D29 (1994) 299. 


\section{FIGURES}

FIG. 1. Comparison of magnetization distributions of spherical clusters and deformed clusters, for a range of inertia ratios $I_{1} / I_{3}$.

FIG. 2. Comparison of average magnetization of mixed- $K$ ensemble with fixed- $K$ ensemble and with the Langevin function, eq. (1).

FIG. 3. Energy levels for locked-spin Hamiltonian as a function of magnetic field, for low $M=1$ states. The spin is tipped by $5^{\circ}$ with respect to the symmetry axis.

FIG. 4. Magnetization distribution of deformed clusters, $I_{1} / I_{3}=2$, with three different spin angles $\theta=0^{\circ}, 45^{\circ}$, and $90^{\circ}$.

FIG. 5. Magnetization distribution of clusters in the anisotropic model, showing the strong coupling limit. Here the spin $(s=1)$ is coupled to the easy axes, with $\lambda=5 \mu B$ and $x=2$. The distribution is close to the result of locked-spin model (dashed line). The distribution is plotted as a function of the rescaled magnetization $\bar{\mu}$.

FIG. 6. Magnetization distribution for the weakly coupled anisotropic Hamiltonian. The spin value is $S=1$, giving three peaks.

FIG. 7. Magnetization distribution for weakly coupled spin to rotor, $\lambda=0.01 k T$. The solid curve shows the distribution in a very weak field, $x=0.005$, and the dashed line shows the distribution in a field $x=0$, somewhat stronger than the anisotropy energy.

FIG. 8. Average magnetization of the anisotropic model for $\lambda=0.01 k T$, as a function of Langevin parameter $x=\mu B / k T$.

FIG. 9. Fit of the low-field adiabatic susceptibility coefficient $a$ in eq. (12) as a function of the anisotropy energy. The temperature is fixed at $T=50$. 







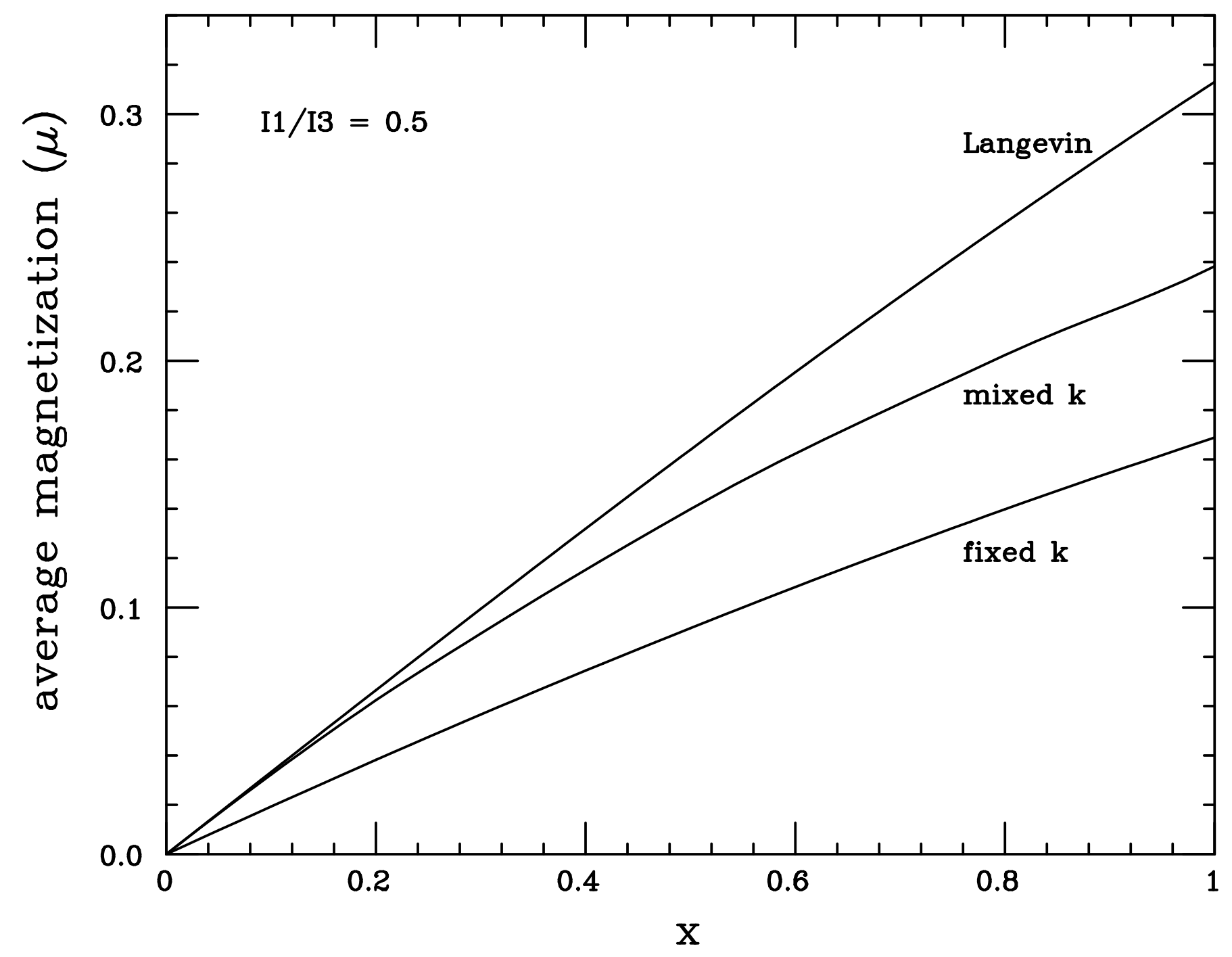




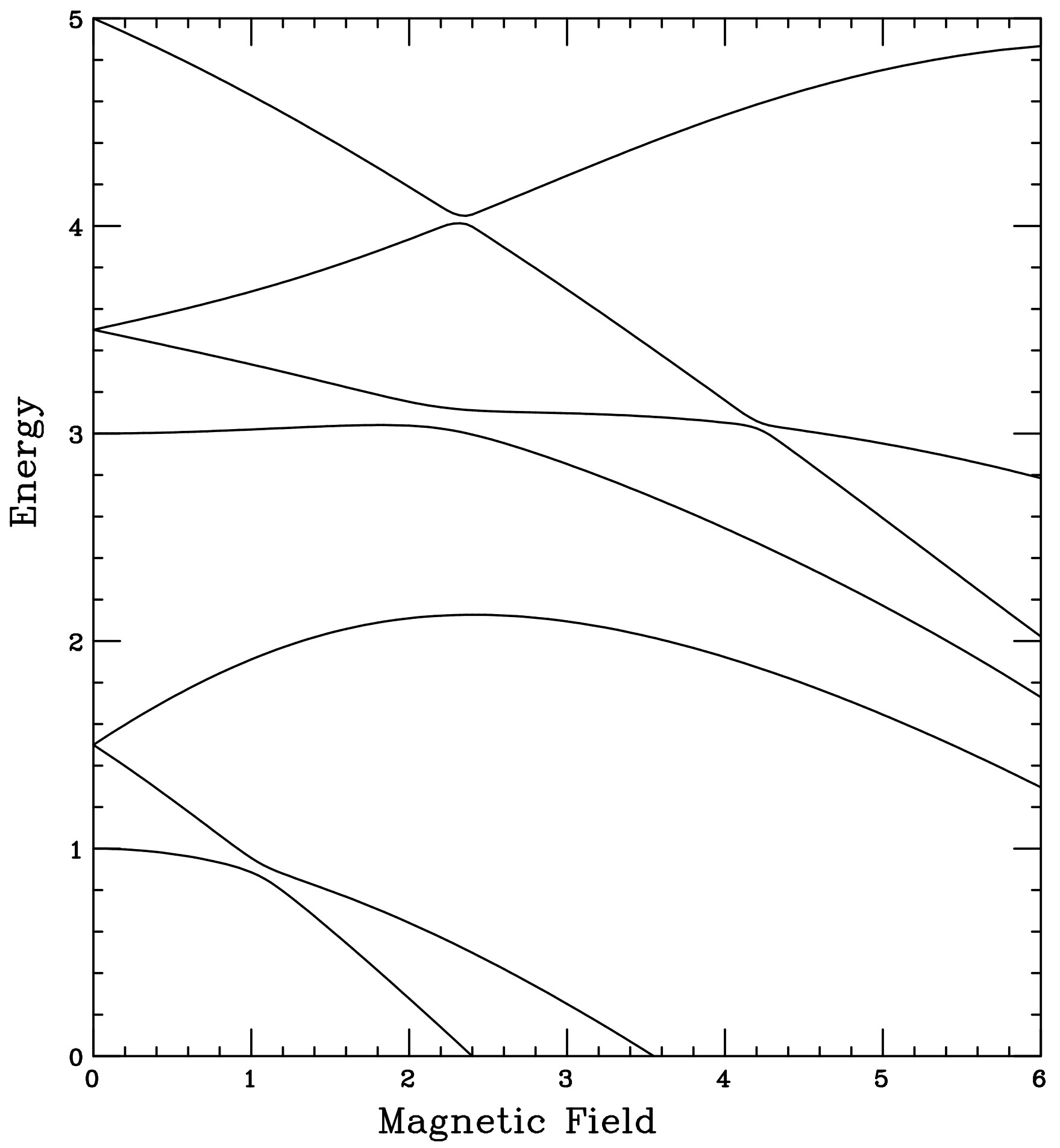




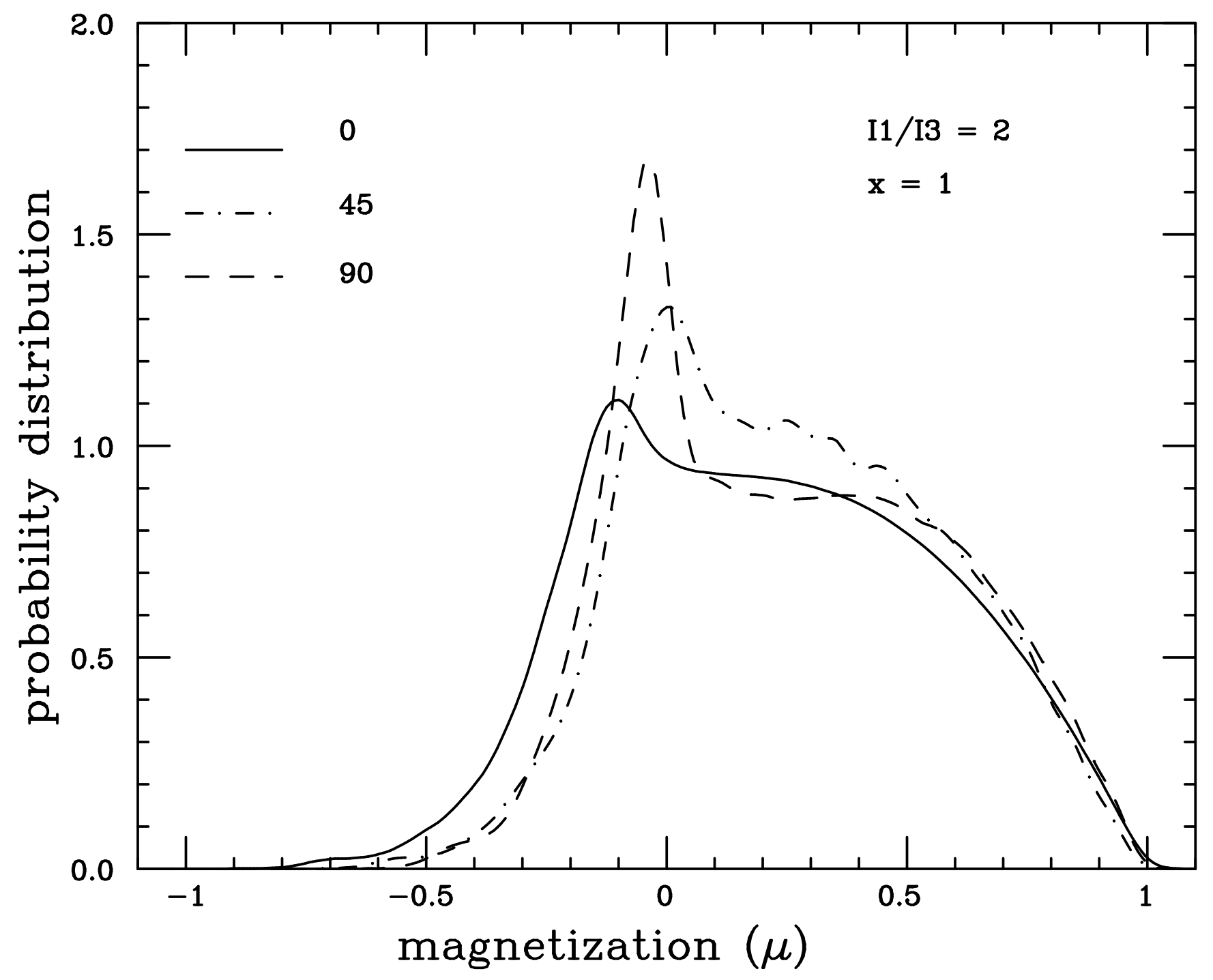









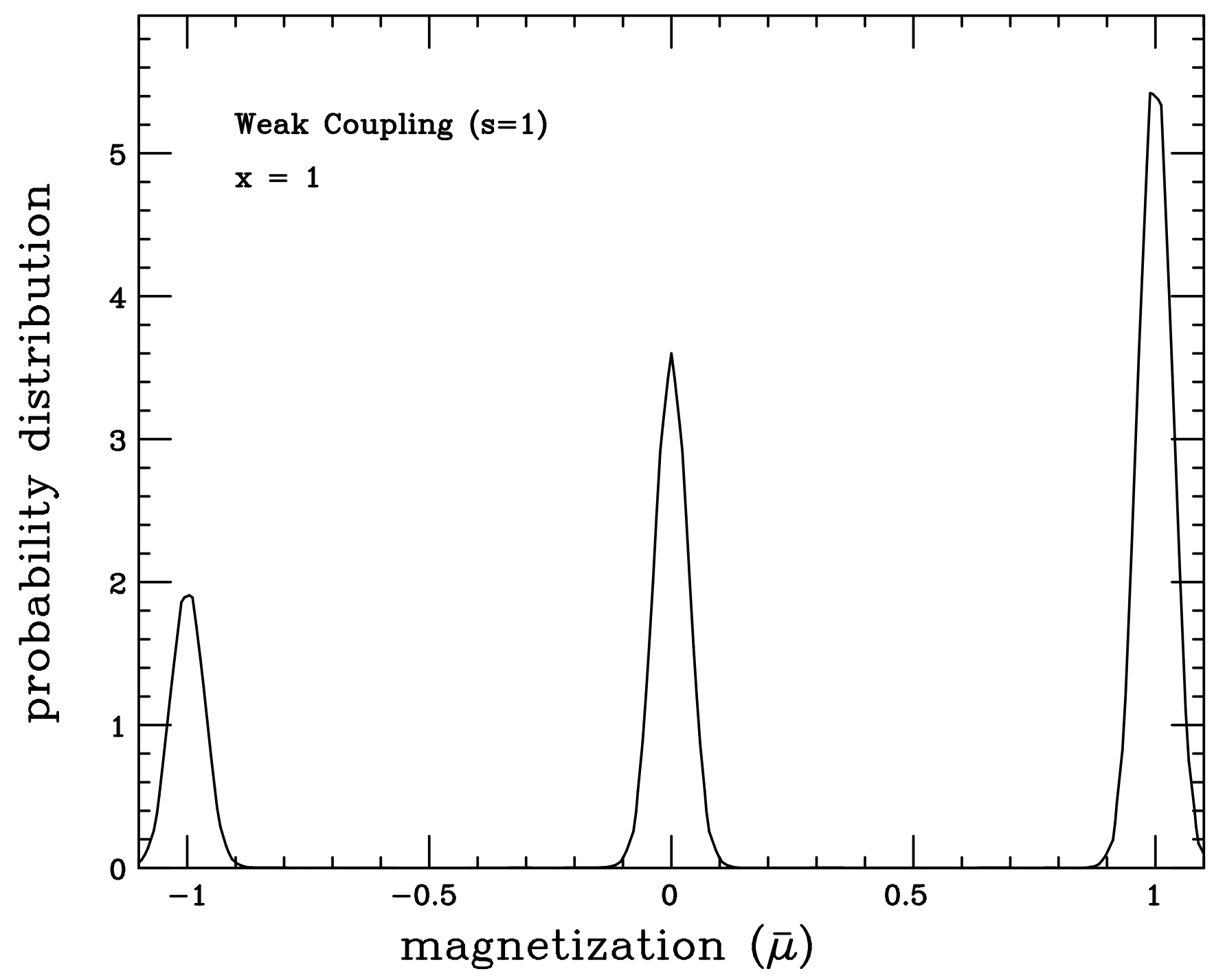




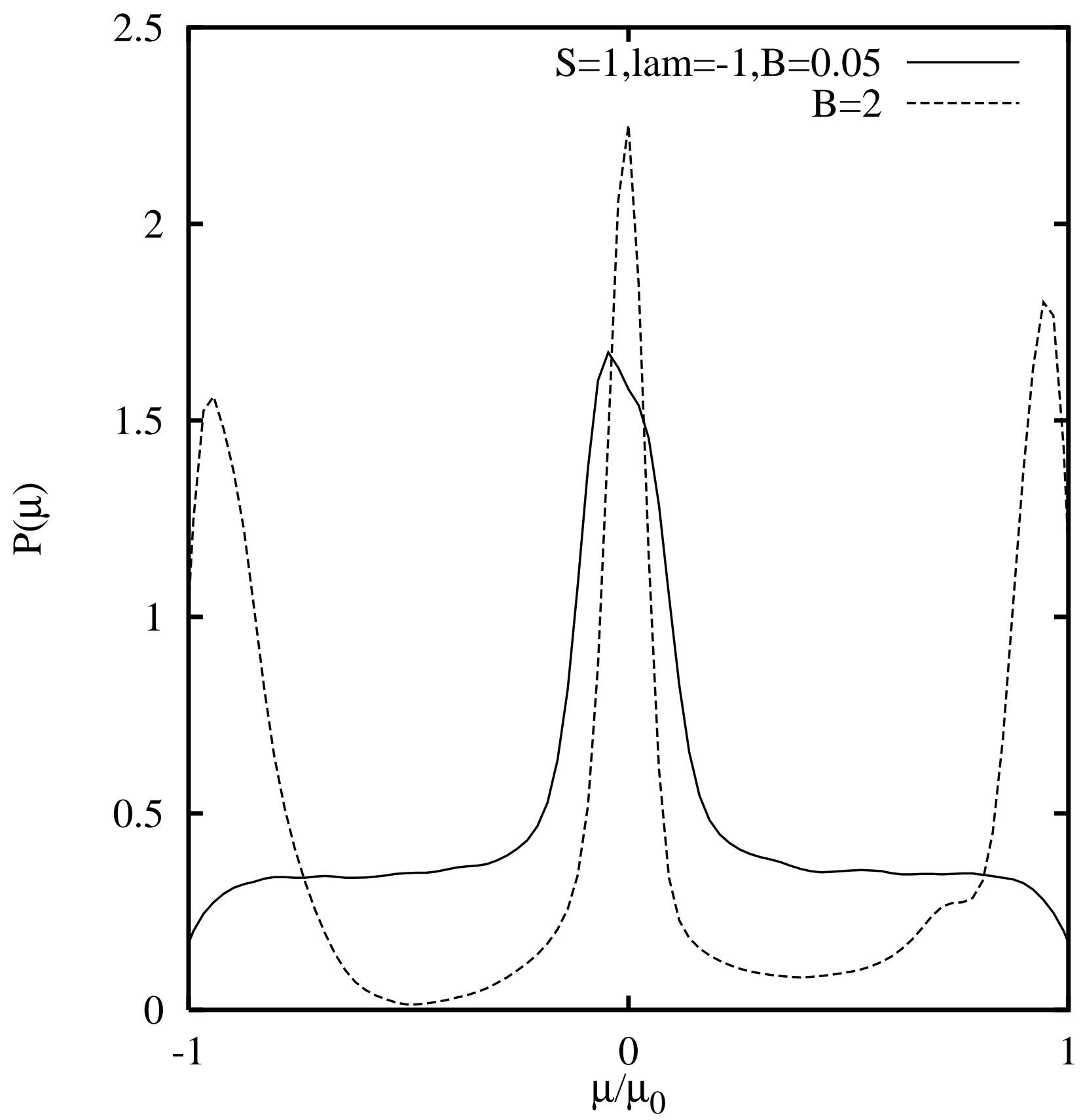











